\title{
Role of superior hypophyseal artery in visual function impairment after paraclinoid carotid artery aneurysm surgery
}

\author{
Tetsuyoshi Horiuchi, MD, ${ }^{1}$ Tetsuya Goto, MD, ${ }^{1}$ Yuichiro Tanaka, MD, ${ }^{2}$ Kunihiko Kodama, MD, ${ }^{1}$ \\ Keiji Tsutsumi, MD, ${ }^{1}$ Kiyoshi Ito, MD, ${ }^{1}$ and Kazuhiro Hongo, MD' \\ 'Department of Neurosurgery, Shinshu University School of Medicine, Matsumoto; and 2Department of Neurosurgery, St.
Marianna University School of Medicine, Kawasaki, Japan
}

\begin{abstract}
OBJECT Although a number of studies have assessed the surgical treatment of paraclinoid-segment carotid artery aneurysms and resulting visual complications, less attention has been given to the results with respect to the superior hypophyseal artery (SHA). The authors evaluated the relationship between the aneurysm, the SHA itself, and postoperative visual function in patients with ruptured and unruptured SHA aneurysms.

METHODS From January 1991 through December 2013, 181 patients with 190 paraclinoid carotid artery aneurysms received treatment at Shinshu University Hospital and its affiliated hospitals. The authors retrospectively analyzed charts, operative records, operative videos, and neuroimaging findings for these patients with or without postoperative visual complications.

RESULTS The authors identified 72 SHA aneurysms in 70 patients (mean age 58 years). Of 69 patients (1 patient died) evaluated, postoperative visual complications occurred in 9 (13.0\%). Although the aneurysm size and SHA sacrifice did not lead to postoperative visual impairment, simultaneous treatment of bilateral aneurysms was a risk factor for postoperative visual complications.
\end{abstract}

CONCLUSIONS Unilateral SHA impairment may be safe (i.e., it may not induce ischemia of the optic pathway) for many, but not all, patients with SHA aneurysm.

http://thejns.org/doi/abs/10.3171/2014.12.JNS141218

KEY WORDS aneurysm; internal carotid artery; superior hypophyseal artery; surgery; vascular disorders

$\Lambda$ CCORDING to the related branching artery or anatomical relationships, there are many classifications of intradural proximal carotid artery aneurysms. In the literature, the term "paraclinoid carotid aneurysm" is often used because it includes all carotid artery aneurysms originating from the internal carotid artery (ICA) between the distal dural ring and the origin of the posterior communicating artery. ${ }^{11,12,21}$ The superior hypophyseal artery (SHA) is well known as a branching artery of paraclinoid carotid artery aneurysms $s^{16,21,22}$ and supplies the pituitary stalk, optic nerve, and chiasm..$^{8,14,18}$ Therefore, sacrifice of the SHA may be a factor for postoperative visual impairment. ${ }^{4,913}$ Although a few case reports have described postoperative visual impairment associated with SHA oc- clusion, ${ }^{4,9,13}$ only small series of SHA aneurysm surgery cases have focused on postoperative visual function and intraoperative SHA status. We report our experience with 72 SHA aneurysms in 70 patients and our analysis of the postoperative visual function associated with the SHA.

\section{Methods}

This study was approved by the institutional review board at the Shinshu University School of Medicine.

\section{Patient Population}

Between January 1991 and December 2013, a total of 181 patients harboring 190 paraclinoid carotid artery an-

ABBREVIATIONS ICA = internal carotid artery; SHA = superior hypophyseal artery; VEP = visual evoked potential.

SUBMITTED June 3, 2014. ACCEPTED December 29, 2014.

INCLUDE WHEN CITING Published online February 20, 2015; DOI: 10.3171/2014.12.JNS141218.

DISCLOSURE The authors report no conflict of interest concerning the materials or methods used in this study or the findings specified in this paper. This study was partly supported by the Japan Society for the Promotion of Science KAKENHI grant no. 25462251 (to T.H). 
eurysms underwent clipping surgery at Shinshu University Hospital and its affiliated hospitals. Patients with paraclinoid carotid artery aneurysms treated with endovascular therapy were excluded from this study. Preoperative and postoperative visual function was evaluated at the bedside and/or by ophthalmological examination. A postoperative visual complication was defined as worsening of visual acuity and/or visual field defect at discharge. A retrospective review of clinical records, neuroimaging findings, and operative videos, including operative records, was conducted to collect information on preoperative symptoms, patient age and sex, aneurysm size and location, surgical approach, and intraoperative SHA findings.

\section{Classification of Paraclinoid Carotid Artery Aneurysms}

Paraclinoid carotid artery aneurysms were classified into 5 groups according to preoperative neuroimaging results: carotid-ophthalmic artery aneurysm, SHA aneurysm, ${ }^{21}$ carotid cave aneurysm, ${ }^{16}$ anterior wall (dorsal) aneurysm, ${ }^{11}$ and others. Both carotid cave and SHA aneurysms projected medially or inferomedially beneath the optic nerve. In the study reported here, SHA aneurysms were preoperatively distinguished from carotid cave aneurysms according to location on the neck of the aneurysm. A carotid cave aneurysm was located below the origin of the ophthalmic artery. ${ }^{16,19}$ Preoperatively, we defined an SHA aneurysm as an aneurysm arising not from the ophthalmic artery but rather from the medial or inferomedial surface of the intradural ICA between the origin of the ophthalmic artery and the posterior communicating artery.

\section{Approach Selection}

Ruptured paraclinoid carotid artery aneurysms were treated via the ipsilateral approach for proximal control of bleeding. For patients with unruptured aneurysms, the ipsilateral or contralateral approach was selected according to preoperative neuroimaging findings..$^{15}$ The affected cervical carotid artery was routinely prepared for proximal control. For some large aneurysms, the suction decompression technique was applied.

\section{Association Between Aneurysm and SHA}

On the basis of operative findings, the origin of the SHA was classified as proximal neck, medial neck, distal neck, aneurysm body, and no relation to the aneurysm (Fig. 1). The final SHA status after clipping was dichotomized into spared and sacrificed.

\section{Visual Evoked Potential Monitoring}

Since December 2004, visual evoked potentials (VEPs) have been used for intraoperative visual function monitoring. ${ }^{9,17}$ Intraoperative VEP monitoring was used in 18 of 32 patients with paraclinoid carotid artery aneurysms, including SHA aneurysms, who have been treated since 2004. When the contralateral approach was used, the SHA related to the aneurysm neck was temporarily occluded (Fig. 2) and changes in VEPs were checked. ${ }^{9}$ If the VEPs did not change after temporary SHA occlusion, complete obliteration took first priority. However, if the VEPs decreased after temporary SHA occlusion, the SHA was spared. ${ }^{9}$

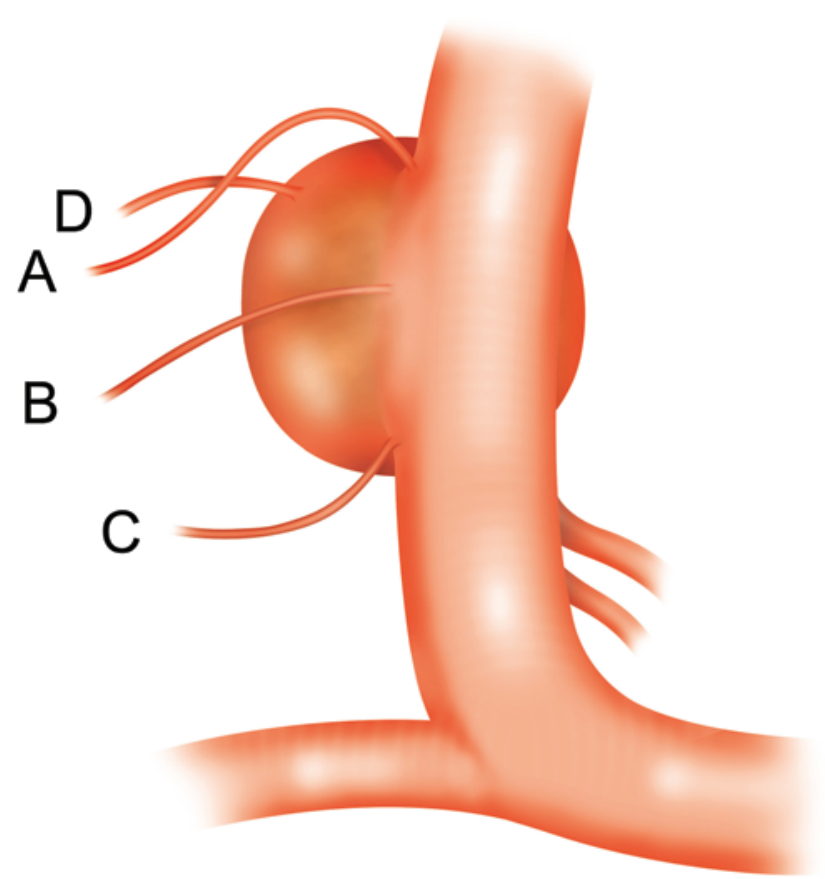

FIG. 1. Illustration showing the relationship between aneurysm and the SHA. The SHA originates from the proximal neck (A), the medial neck (B), the distal neck (C), and the aneurysm body (D). Copyright Tetsuyoshi Horiuchi. Published with permission.

\section{Statistical Analysis}

Statistical analyses were performed with PASW Statistics version 21 (SPSS). Variables were compared among the groups divided according to visual outcome. The unpaired t-test and the Pearson chi-square test or Fisher exact test were used to compare continuous and categorical variables, respectively. In all analyses, $\mathrm{p}<0.05$ was considered to indicate statistical significance.

\section{Results}

The 72 SHA aneurysms (37.9\%) in 70 patients (38.7\%) (12 men and 58 women) were treated with clipping. Table 1 shows the baseline characteristics of the 70 patients. Mean patient age was 58.0 years (range $26-87$ years). The 72 aneurysms ranged in size from 2 to $23 \mathrm{~mm}$. Preoperative presentation was subarachnoid hemorrhage for 17 patients, mass effect for 3 , and incidental identification for 50. For 2 patients with bilateral SHA aneurysms and 1 patient with a left SHA aneurysm and a right carotid cave aneurysm, clipping surgery for each aneurysm was performed at the same time through one frontotemporal craniotomy. Of the 70 patients, 1 died postoperatively as a result of initial brain damage caused by subarachnoid hemorrhage.

\section{Postoperative Visual Function}

Postoperative visual functions were evaluated for the 69 surviving patients. For 1 patient, visual acuity improved postoperatively (Table 1), and for 9 patients (13.0\%), visual status deteriorated postoperatively (Tables 1 and 2). Postoperative visual impairment was not affected by patient 

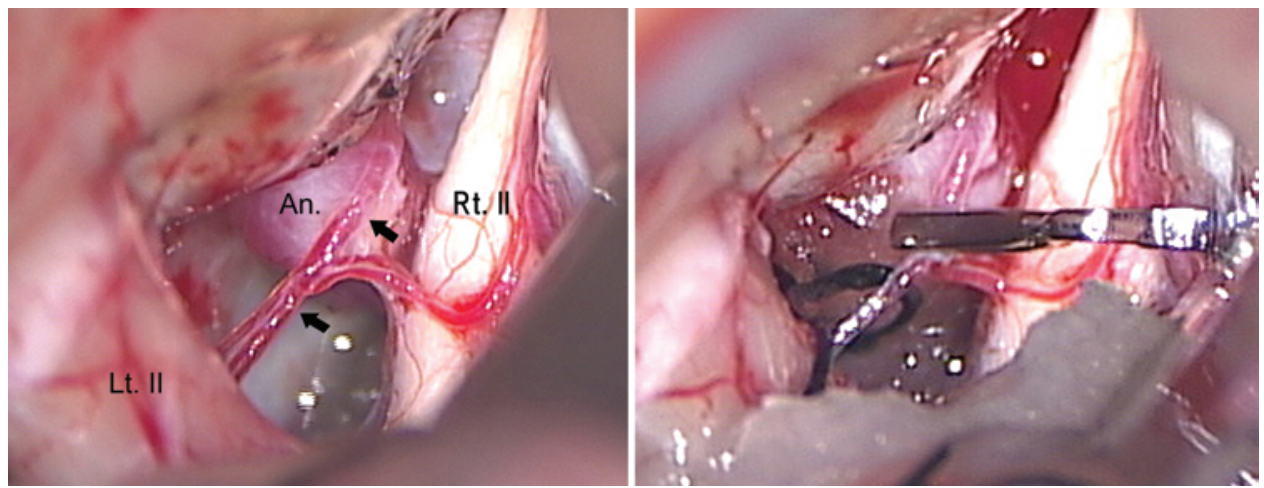

FIG. 2. Intraoperative photographs showing temporary occlusion of the SHA (arrows) before (left) and after (right) application of a microclip in a right-sided SHA aneurysm through a contralateral approach. An. = aneurysm; Lt. II = left optic nerve; Rt. II = right optic nerve.

age, sex, preoperative symptoms, and aneurysm side (Table 2). Aneurysm size did not differ significantly between groups with and without visual impairment, although the trend was toward larger aneurysms in the group that experienced visual impairment $(\mathrm{p}=0.080)$.

\section{Association Between Surgery and Visual Function}

The surgical approach was not associated with visual impairment. A significant factor for visual impairment appears to be simultaneous clipping for bilateral paraclinoid carotid artery aneurysms $(\mathrm{p}=0.002)$. Vision was impaired postoperatively for all 3 patients who underwent clipping of bilateral aneurysms in 1 stage; for these 3 patients, VEP monitoring was not available at the time of surgery. In 2 patients, bilateral SHAs were sacrificed during aneurysm clipping ( 1 of these patients is described in Case 1 [see Illustrative Cases]) and bilateral visual impairment developed after surgery.

\section{Association Between SHA and Visual Function}

The SHA was identified in 45 (62.5\%) of 72 aneurysms. SHA origin was related to the aneurysm neck or dome in $37(82.2 \%)$ of these 45 aneurysms, and the SHA was not related to the aneurysm in the operative field in the other 8 patients. We did not identify or determine the origin of the SHA in some patients because dense subarachnoid hemorrhage and/or the optic apparatus restricted observation, and exploration of the entire neck was obscured by the lesion itself or by the ICA. The relationships between the aneurysm and the origin of the SHA are presented in Tables 1 and 2. The most commonly affected origin of the SHA was the proximal neck. No significant difference in SHA origin was observed between patients in groups with and without visual impairment. The status of the SHA after clipping was assessed for $44(97.8 \%)$ of 45 aneurysms. The SHA was sacrificed in $25(56.8 \%)$ of these 44 aneurysms. Bilateral SHAs were obliterated in 1 patient (Case 1). Of 24 patients, 2 complained of visual impairment after surgery. One of these patients is described in Case 1; the other patient, who had a left SHA aneurysm and a right carotid cave aneurysm, underwent clipping surgery for each aneurysm simultaneously through a right-sided craniotomy. For this patient, the left aneurysm and the SHA
TABLE 1. Baseline characteristics for 70 patients with 72 SHA aneurysms

\begin{tabular}{|c|c|}
\hline Characteristic & Value $(\%)^{*}$ \\
\hline \multicolumn{2}{|l|}{ Sex } \\
\hline M & 12 (17.1) \\
\hline $\mathrm{F}$ & $58(82.9)$ \\
\hline \multicolumn{2}{|l|}{ Age (yrs) } \\
\hline Mean & 58.0 \\
\hline Range & $26-87$ \\
\hline \multicolumn{2}{|l|}{ Aneurysm location } \\
\hline Rt & $32(44.4)$ \\
\hline $\mathrm{Lt}$ & $38(52.8)$ \\
\hline Bilat & $2(2.8)$ \\
\hline \multicolumn{2}{|l|}{ Surgical approach } \\
\hline Ipsilat & $46(63.9)$ \\
\hline Contralat & $26(36.1)$ \\
\hline \multicolumn{2}{|c|}{ Aneurysm size (mm) } \\
\hline Mean & 6.7 \\
\hline Range & $2-23$ \\
\hline \multicolumn{2}{|c|}{ Postop visual function } \\
\hline Improved & $1(1.4)$ \\
\hline Unchanged & $59(84.3)$ \\
\hline Worsened & $9(12.9)$ \\
\hline Undetermined & $1(1.4)$ \\
\hline \multicolumn{2}{|l|}{ SHA origin } \\
\hline Proximal neck & $23(31.9)$ \\
\hline Medial neck & $4(5.6)$ \\
\hline Distal neck & $4(5.6)$ \\
\hline Body & $6(8.3)$ \\
\hline No relation & $8(11.1)$ \\
\hline Undetermined & $27(37.5)$ \\
\hline \multicolumn{2}{|l|}{ SHA preservation } \\
\hline Spared & $19(26.4)$ \\
\hline Sacrificed & $25(34.7)$ \\
\hline Undetermined & $28(38.9)$ \\
\hline
\end{tabular}

* Values represent number (\%) of patients or aneurysms. 
TABLE 2. Clinical relationships between favorable and unfavorable visual function outcome for 69 patients after clipping of SHA aneurysms

\begin{tabular}{|c|c|c|c|}
\hline \multirow[b]{2}{*}{ Characteristic } & \multicolumn{2}{|c|}{ Postop Visual Function (\%) } & \multirow[b]{2}{*}{$\begin{array}{c}p \\
\text { Value }\end{array}$} \\
\hline & $\begin{array}{c}\text { w/o } \\
\text { Impairment }\end{array}$ & $\begin{array}{c}\text { w/ } \\
\text { Impairment }\end{array}$ & \\
\hline Patients, no. & $60(87.0)$ & $9(13.0)$ & \\
\hline Mean age \pm SD (yrs) & $57.2 \pm 10.9$ & $61.3 \pm 7.1$ & 0.276 \\
\hline Size of aneurysm $\pm \mathrm{SD}(\mathrm{mm})$ & $6.1 \pm 4.9$ & $9.2 \pm 4.2$ & 0.080 \\
\hline Sex & & & 1.000 \\
\hline M & $11(18.3)$ & $1(11.1)$ & \\
\hline $\mathrm{F}$ & $49(81.7)$ & $8(88.9)$ & \\
\hline Preop symptom & & & 0.411 \\
\hline Subarachnoid hemorrhage & $15(25.0)$ & $1(11.1)$ & \\
\hline Mass effect & $2(3.3)$ & $1(11.1)$ & \\
\hline Incidental finding & $43(71.7)$ & $7(77.8)$ & \\
\hline Aneurysm location & & & 0.500 \\
\hline $\mathrm{Rt}$ & $28(46.7)$ & $3(33.3)$ & \\
\hline Lt & $32(53.3)$ & $6(66.7)$ & \\
\hline Surgical approach & & & 0.147 \\
\hline Ipsilat & $37(61.7)$ & $8(88.9)$ & \\
\hline Contralat & $23(38.3)$ & $1(11.1)$ & \\
\hline $\begin{array}{l}\text { Bilat aneurysms treated simul- } \\
\text { taneously }\end{array}$ & & & 0.002 \\
\hline Yes & 0 & $3(33.3)$ & \\
\hline No & $60(100)$ & $6(66.7)$ & \\
\hline SHA origin & & & 0.292 \\
\hline Proximal neck & $20(33.3)$ & $2(22.2)$ & \\
\hline Medial neck & $3(5.0)$ & $1(11.1)$ & \\
\hline Distal neck & $4(6.7)$ & 0 & \\
\hline Body & $6(10.0)$ & 0 & \\
\hline No relation & $8(13.3)$ & 0 & \\
\hline Undetermined & $19(31.7)$ & $6(66.7)$ & \\
\hline SHA preservation & & & 0.150 \\
\hline Spared & $18(30.0)$ & $1(11.1)$ & \\
\hline Sacrificed & $22(36.7)$ & $2(22.2)$ & \\
\hline Undetermined & $20(33.3)$ & $6(66.7)$ & \\
\hline
\end{tabular}

were occluded with the clip, and vision was disturbed in both eyes postoperatively. None of the 22 patients with a unilateral aneurysm experienced postoperative visual impairment after unilateral SHA sacrifice. SHA sacrifice did not affect the occurrence of the visual impairment in this study $(\mathrm{p}=0.150)$.

\section{VEP Monitoring}

VEP monitoring was performed in 6 patients with SHA aneurysms. Temporary occlusion of the SHA through the contralateral approach was applied in 3 patients (Fig. 2). Significant VEP change was noted in 1 patient, ${ }^{9}$ and no change was noted in 2 patients. Surgical manipulation attenuated the VEPs in 1 patient with postoperative worsening of visual acuity (Case 2).

\section{Illustrative Cases \\ Case 1}

A 74-year-old woman received care at another hospital for a subarachnoid hemorrhage 2 weeks before coming to Shinshu University Hospital. She also had a ruptured left SHA aneurysm and an unruptured right SHA aneurysm with middle cerebral artery aneurysms (Fig. 3). Preoperatively, no visual disturbance was noted. After the left cervical ICA was secured, a left frontotemporal craniotomy was performed. At that time, VEP monitoring was not available. The right SHA aneurysm was visible in the prechiasmatic cistern, and the right SHA originated from the proximal neck (Fig. 3). The right-sided aneurysm with SHA was clipped using a curved clip. The left anterior clinoid process was removed, and the left SHA aneurysm was exposed. The left SHA was branched from the medial neck of the aneurysm. The left-sided aneurysm including the SHA was obliterated using 2 ring clips (Fig. 3). Postoperatively, the patient complained of visual disturbance in both eyes. Detailed ophthalmological examination was not performed because of the patient's poor general condition. The visual symptoms gradually improved, and the patient was transferred to a rehabilitation hospital.

\section{Case 2}

A 55-year-old woman with left visual disturbance received a diagnosis of a large left SHA aneurysm (Fig. 4). Her visual acuity in the left eye was 20/100. With VEP monitoring, the aneurysm was obliterated through left frontotemporal craniotomy. No SHA associated with the aneurysm was found in the operative field (Fig. 4). The aneurysm was well clipped by using a retrograde suction decompression technique (Fig. 4). The left VEPs were attenuated during aneurysm dissection at the time of suction decompression. Postoperatively, the left visual acuity decreased to $20 / 300$.

\section{Discussion}

To the best of our knowledge, this study is the largest series to consider postoperative visual function and detailed intraoperative SHA findings. Although unilateral SHA sacrifice rarely induces visual impairment, simultaneous bilateral SHA occlusion is a factor for visual disturbance.

\section{Paraclinoid Carotid Artery Aneurysm and Visual Function}

Because paraclinoid carotid artery aneurysms are in close proximity to the visual apparatus, they often induce visual disturbance, and treatment of these lesions can produce visual symptoms. ${ }^{3,5,6,10}$ Visual complications remain a major concern even for the most experienced vascular surgeons. Visual deficits associated with clipping surgery for paraclinoid carotid artery aneurysm can be caused by vascular compromise, manipulation of the optic nerve, thermogenic effects from high-speed drilling of the clinoid process, or other unknown causes. ${ }^{3,5,6,10,19,20}$ For the patient in Case 2, surgical manipulation was the most likely cause of the postoperative visual worsening because VEPs were attenuated during the suction decompression procedure.

In 1981, Ferguson and Drake ${ }^{5}$ reported on a series of 100 surgical carotid-ophthalmic aneurysm cases, of which 


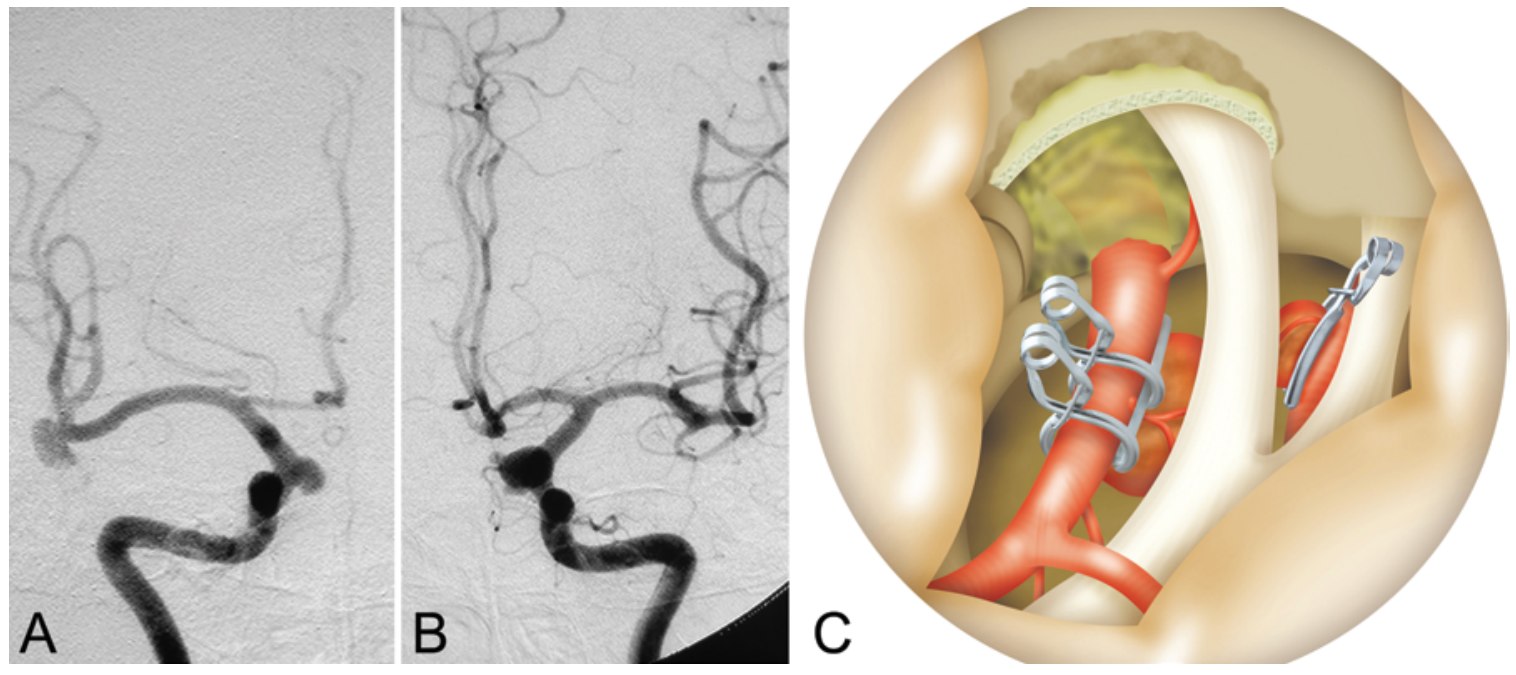

FIG. 3. Anteroposterior projections of preoperative right (A) and left (B) carotid artery angiograms revealing bilateral SHA aneurysms and a right middle cerebral artery aneurysm. Schematic drawing (C) showing the clipped bilateral SHA aneurysms through the left craniotomy. The left and right SHAs originated from the medial and proximal necks, respectively. Both SHAs were occluded by clips. Schematic drawing: Copyright Tetsuyoshi Horiuchi. Published with permission.

32 of the 100 patients had visual abnormalities at the time of presentation. In 1990, Day ${ }^{3}$ reported preoperative visual deficits in $29(36.3 \%)$ of 80 patients with ophthalmic segment aneurysms and postoperative deterioration of visual status in $6(7.5 \%)$ of 80 patients. Of these 6 patients, visual field defects occurred in 2 patients, visual acuity diminution in 1, and transient palsies of the sixth cranial nerve in 3. In 2009, Fulkerson et al. ${ }^{6}$ reported that new visual loss occurred in 6 (4.8\%) of 126 ophthalmic aneurysm patients who underwent craniotomy. In contrast, Barami et al. ${ }^{1}$ reported that vision impairment associated with clipping for paraclinoid carotid artery aneurysms might comprise up to $20 \%$ of cases at 6-month follow-up examination. In our series, postoperative visual deterioration occurred in $9(13.0 \%)$ of 69 patients. No significant differences in aneurysm size and side, preoperative symptoms, or surgi-
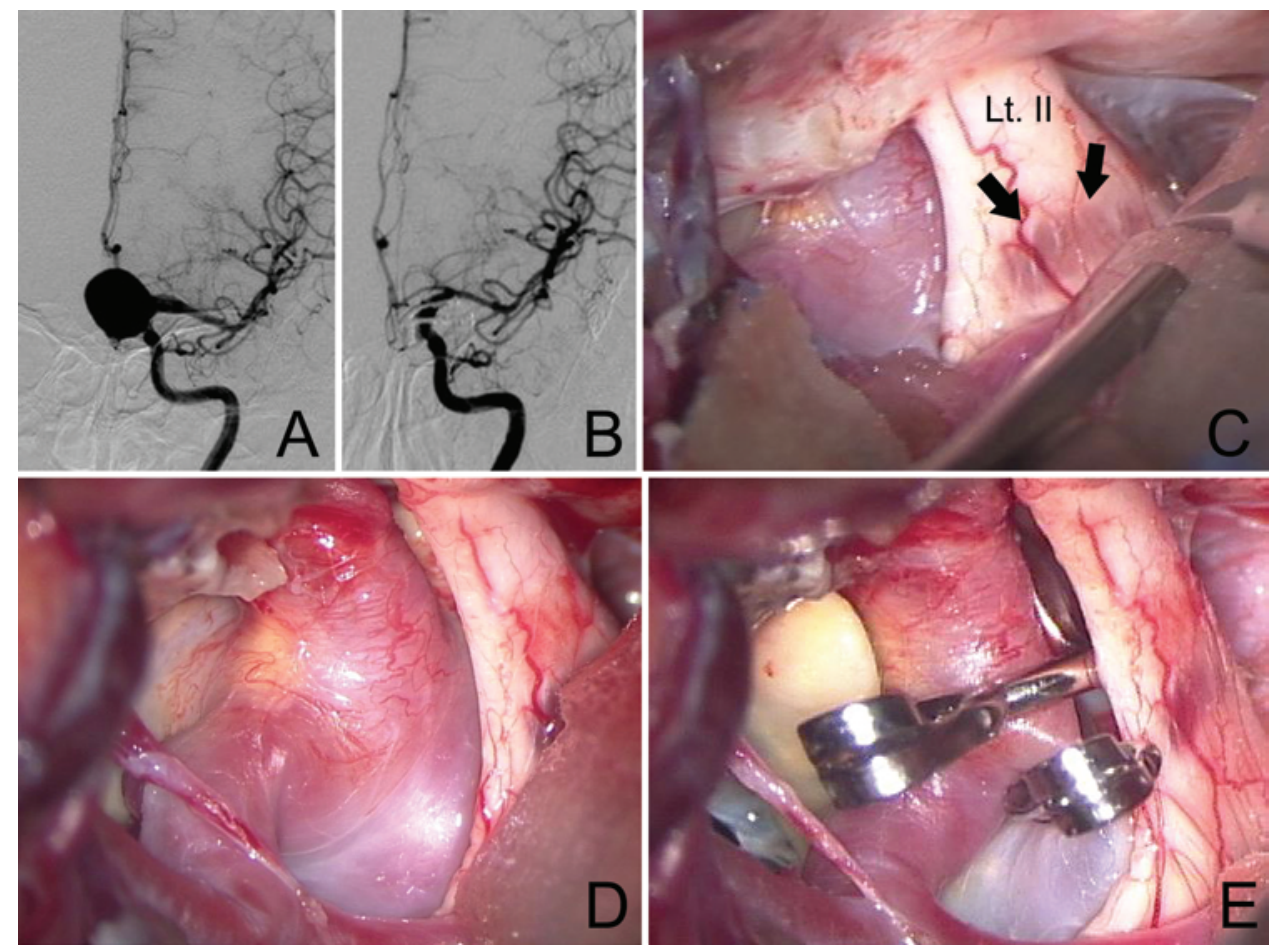

FIG. 4. Anteroposterior projections of serial left carotid artery angiograms obtained preoperatively (A) and postoperatively (B) showing that the SHA aneurysm was successfully clipped. Intraoperative photographs (C-E) through the left pterional approach showing the indentation of the left optic nerve (Lt. II) (arrows) by the aneurysm. There was no SHA associated with the aneurysm neck, and 2 clips were applied to the aneurysm. 
cal approach were observed between the groups with and without visual impairment. The simultaneous treatment of bilateral aneurysms was a factor associated with postoperative visual deterioration. This finding suggests that despite improvements in microsurgical techniques and instruments, surgical manipulation of the bilateral optic apparatus including SHAs is dangerous.

\section{SHA and Visual Function}

Although the SHA may play a major role in visual function because it supplies the optic nerve and chiasm, few have studied postoperative visual function after SHA sacrifice. SHAs comprise a group of 1-5 small branches arising from the medial or inferomedial surface of the ICA between the distal dural ring and the origin of the posterior communicating artery. ${ }^{8,14,18}$ SHAs are also well known as aneurysm sites. Day ${ }^{3}$ reported that SHA preservation should be attempted in all patients but that it is not always possible because this artery is small and easily obliterated by fenestrated clips. Because there are circumferential anastomoses of the SHA,${ }^{7,8,18}$ obliteration of the SHA will not cause visual disturbance..$^{2,3,15,21}$ Our study also showed that SHA sacrifice was not a factor for postoperative visual impairment. Unilateral SHA sacrifice associated with aneurysm surgery did not induce visual disturbance. However, postoperative visual complications may be caused by ischemia associated with SHA sacrifice. ${ }^{3-6,9,10,20}$ In 2007 , Goto et al. ${ }^{9}$ reported that the temporary occlusion of the SHA in the contralateral approach for SHA aneurysms attenuated VEPs and that the preservation of the SHA avoided vision complications. This report indicates a possibility that a unilateral SHA supplies the bilateral optic apparatus or that no circumferential anastomosis is present between right and left SHAs in rare cases. Recently, Johnson et al. ${ }^{13}$ reported a case of optic pathway infarction caused by SHA occlusion after aneurysm embolization with Onyx HD 500 (eV3/Covidien). This complication can be explained by extension of the embolic material to the microvessels where it occluded the collateral pathway of the SHA. In 2012, El Refaee et al. ${ }^{4}$ reported a case of postoperative bilateral visual field defects associated with SHA occlusion. In that case, indocyanine green angiography demonstrated retrograde filling of the occluded SHA. This finding indicated that there was some collateral blood flow through the anastomosis; however, it was unclear whether the retrograde blood flow would be sufficient. In previous anatomical studies, ${ }^{7,8,18}$ unilateral variation of the SHA has not been reported. If there is no circumferential anastomosis between the right and left SHAs in some patients, unilateral SHA occlusion must cause ischemia of the optic nerve and/or chiasm, resulting in postoperative visual disturbance.

In our study, the simultaneous treatment of bilateral aneurysms was a significant risk factor for postoperative visual complications. This finding suggests that arterial injury of bilateral SHAs associated with aneurysms would result in ischemia of the optic nerve and chiasm. Therefore, special attention should be paid when bilateral paraclinoid carotid artery aneurysms are treated simultaneously. Even with unilateral treatment, a few patients will experience postoperative visual complications associated with SHA ischemia., ${ }^{4,13}$ Because VEP monitoring may be useful for detecting ischemia associated with SHA occlusion, ${ }^{9}$ we recommend use of VEP monitoring to avoid visual complications.

\section{Relationship Between the Origin of the SHA and Aneurysm}

The anatomical relationship between SHA origin and SHA aneurysm is poorly understood because it is usually difficult to evaluate the SHAs on preoperative angiograms and explore the entire neck hidden by the optic nerve and ICA. Previous reports have shown that SHAs originated from the proximal neck, distal neck, medial neck, and aneurysm body. ${ }^{3,4,9,22}$ In our study, most aneurysms involved SHA branching from the proximal neck. No significant differences in SHA origins were noticed with regard to postoperative visual complications.

\section{Conclusions}

To our knowledge, this is the largest series reported to date concerning postoperative visual complications associated with intraoperative SHA status. Unilateral SHA sacrifice may be safe for many, but not all, patients with SHA aneurysm. Special attention should be paid to simultaneous treatment for bilateral paraclinoid carotid artery aneurysms.

Use of advanced microsurgical techniques and intraoperative VEP monitoring will substantially improve visual outcomes after microsurgery for paraclinoid carotid artery aneurysm, especially in patients with SHA aneurysms.

\section{References}

1. Barami K, Hernandez VS, Diaz FG, Guthikonda M: Paraclinoid carotid aneurysms: surgical management, complications, and outcome based on a new classification scheme. Skull Base 13:31-41, 2003

2. Batjer HH, Kopitnik TA, Giller CA, Samson DS: Surgery for paraclinoidal carotid artery aneurysms. J Neurosurg 80:650-658, 1994

3. Day AL: Aneurysms of the ophthalmic segment. A clinical and anatomical analysis. J Neurosurg 72:677-691, 1990

4. El Refaee EA, Baldauf J, Balau V, Rosenstengel C, Schroeder $\mathrm{H}$ : Is it safe to sacrifice the superior hypophyseal artery in aneurysm clipping? A report of two cases. J Neurol Surg A Cent Eur Neurosurg 74 Suppl 1:e255-e260, 2013

5. Ferguson GG, Drake CG: Carotid-ophthalmic aneurysms: visual abnormalities in 32 patients and the results of treatment. Surg Neurol 16:1-8, 1981

6. Fulkerson DH, Horner TG, Payner TD, Leipzig TJ, Scott JA, DeNardo AJ, et al: Results, outcomes, and follow-up of remnants in the treatment of ophthalmic aneurysms: a 16-year experience of a combined neurosurgical and endovascular team. Neurosurgery 64:218-230, 2009

7. Gibo H, Kobayashi S, Kyoshima K, Hokama M: Microsurgical anatomy of the arteries of the pituitary stalk and gland as viewed from above. Acta Neurochir (Wien) 90:60-66, 1988

8. Gibo H, Lenkey C, Rhoton AL Jr: Microsurgical anatomy of the supraclinoid portion of the internal carotid artery. J Neurosurg 55:560-574, 1981

9. Goto T, Tanaka Y, Kodama K, Kusano Y, Sakai K, Hongo K: Loss of visual evoked potential following temporary occlusion of the superior hypophyseal artery during aneurysm clip placement surgery. Case report. J Neurosurg 107:865-867, 2007

10. Heros RC, Nelson PB, Ojemann RG, Crowell RM, DeBrun $\mathrm{G}$ : Large and giant paraclinoid aneurysms: surgical tech- 
niques, complications, and results. Neurosurgery 12:153163,1983

11. Horiuchi T, Kusano Y, Yako T, Murata T, Kakizawa Y, Hongo K: Ruptured anterior paraclinoid aneurysms. Neurosurg Rev 34:49-55, 2011

12. Horiuchi T, Tanaka Y, Kusano Y, Yako T, Sasaki T, Hongo $\mathrm{K}$ : Relationship between the ophthalmic artery and the dural ring of the internal carotid artery. Clinical article. J Neurosurg 111:119-123, 2009 (Erratum in J Neurosurg 111:193, 2009)

13. Johnson JN, Elhammady M, Post J, Pasol J, Ebersole K, Aziz-Sultan MA: Optic pathway infarct after Onyx HD 500 aneurysm embolization: visual pathway ischemia from superior hypophyseal artery occlusion. J Neurointerv Surg 6:e47, 2014

14. Joo W, Funaki T, Yoshioka F, Rhoton AL Jr: Microsurgical anatomy of the carotid cave. Neurosurgery 70 (2 Suppl Operative):300-312, 2012

15. Kakizawa Y, Tanaka Y, Orz Y, Iwashita T, Hongo K, Kobayashi S: Parameters for contralateral approach to ophthalmic segment aneurysms of the internal carotid artery. Neurosurgery 47:1130-1137, 2000

16. Kobayashi S, Kyoshima K, Gibo H, Hegde SA, Takemae T, Sugita K: Carotid cave aneurysms of the internal carotid artery. J Neurosurg 70:216-221, 1989

17. Kodama K, Goto T, Sato A, Sakai K, Tanaka Y, Hongo K: Standard and limitation of intraoperative monitoring of the visual evoked potential. Acta Neurochir (Wien) 152:643648,2010

18. Krisht AF, Barrow DL, Barnett DW, Bonner GD, Shengalaia $\mathrm{G}$ : The microsurgical anatomy of the superior hypophyseal artery. Neurosurgery 35:899-903, 1994
19. Kumon Y, Sakaki S, Kohno K, Ohta S, Ohue S, Oka Y: Asymptomatic, unruptured carotid-ophthalmic artery aneurysms: angiographical differentiation of each type, operative results, and indications. Surg Neurol 48:465-472, 1997

20. Rizzo JF III: Visual loss after neurosurgical repair of paraclinoid aneurysms. Ophthalmology 102:905-910, 1995

21. Tanaka Y, Hongo K, Tada T, Nagashima H, Horiuchi T, Goto $\mathrm{T}$, et al: Radiometric analysis of paraclinoid carotid artery aneurysms. J Neurosurg 96:649-653, 2002

22. Yapor WY, Crowell RM: Superior hypophyseal artery aneurysm. Report of two cases. J Neurosurg 74:501-503, 1991

\section{Author Contributions}

Conception and design: Horiuchi. Acquisition of data: Horiuchi, Goto, Tanaka, Kodama, Tsutsumi. Analysis and interpretation of data: Horiuchi, Goto, Tanaka, Ito. Drafting the article: Horiuchi. Critically revising the article: Tanaka, Hongo. Reviewed submitted version of manuscript: Goto, Tanaka, Kodama, Tsutsumi, Ito. Approved the final version of the manuscript on behalf of all authors: Horiuchi. Statistical analysis: Horiuchi. Administrative/ technical/material support: Goto, Kodama, Tsutsumi, Ito. Study supervision: Hongo.

\section{Correspondence}

Tetsuyoshi Horiuchi, Department of Neurosurgery, Shinshu University School of Medicine, 3-1-1 Asahi, Matsumoto 3908621, Japan. email: tetuyosi@shinshu-u.ac.jp. 